(c) 2018 - ISSN 1807-2577

\title{
Análise do perfil dos pacientes com deficiência internados no Instituto de Saúde da Criança do Amazonas
}

\author{
Profile analysis of disabled patients in the Amazon Child Health Institute
}

\author{
Sara Maria Araujo do NASCIMENTOa* (i), Gimol Benchimol de RESENDE PRESTES ${ }^{a}$ (1), \\ Eliane de Oliveira Aranha RIBEIROa ${ }^{(0)}$, Keuly Sousa SOARES ${ }^{\circledR}$, \\ Aréli Morais de Araújo ALENCAR ${ }^{\mathrm{a}}$ (D) \\ aUEA - Universidade do Estado do Amazonas, Manaus, AM, Brasil
}

\begin{abstract}
Como citar: Nascimento SMA, Resende Prestes GB, Ribeiro EOA, Soares KS, Alencar AMA. Análise do perfil dos pacientes com deficiência internados no Instituto de Saúde da Criança do Amazonas. Rev Odontol UNESP. 2020;49:e20200062. https://doi.org/10.1590/1807-2577.06220
\end{abstract}

\begin{abstract}
Resumo
Introdução: Pessoas com Deficiência (PcD) são aquelas que possuem alteração física, intelectual, social ou emocional. Podem ter impedimentos que duram um curto período de tempo ou por toda sua vida. Objetivo: Descrever o perfil clínico dos pacientes com deficiência internados no Instituto de Saúde da Criança do Amazonas (ICAM). Material e método: Foram analisados 34 pacientes e coletados dados, como gênero, faixa etária, procedência, patologia de base e condição de saúde bucal. Os dados obtidos foram organizados e tabulados no Microsoft Excel 2013. Resultado: Na distribuição por gênero, verificou-se $70 \%$ do gênero masculino (mais prevalente) e 30\% do feminino. Quanto à faixa etária, a idade predominante foi 8 anos. A maioria dos pacientes (60\%) era do interior do Amazonas e 40\% eram da capital. No que diz respeito à patologia, a Doença Neurológica teve prevalência em $44 \%$ dos casos, seguida das seguintes patologias: Síndrome de Down 18\%, Transtorno de Espectro Autista 12\%, Mielomeningocele 12\%, Surdez 3\%, Diabetes 3\%, Síndrome de Prader Willi 3\%, Síndrome Moebius 3\% e Síndrome de Lennox 3\%. Na condição bucal, $44 \%$ da amostra teve condição precária, 32\% deficiente e $24 \%$ satisfatória. Conclusão: A maioria das crianças era do gênero masculino, faixa etária de 8 anos, procedentes do interior do Amazonas, com deficiência neurológica e condição bucal precária, sugerindo que a presença do cirurgião-dentista, compondo a equipe multidisciplinar hospitalar, auxilia a traçar um plano de tratamento preventivo e curativo adequado a esses indivíduos.
\end{abstract}

Descritores: Pessoas com deficiência; Odontologia Hospitalar; condição bucal.

\begin{abstract}
Introduction: Disabled people is anybody that has physical, intellectual, social or emotional alteration. They can have impediments that last for a short period of time or for the entire life. Objective: Describe the clinical profile of disabled patients admitted to Instituto de Saúde da Criança do Amazonas (ICAM). Material and method: Thirty-four patients were analyzed, data such as gender, age, origin, basis pathology and oral health condition were collected. The data obtained were organized and tabulated in Microsoft Excel 2013. Result: There was a prevalence of males with $70 \%$ and $30 \%$ females. The age group had a predominance of 8 years. Most of the patients 60\% were from Amazonas inland cities and $40 \%$ from the capital. Regarding to the pathology, Neurological Disease was prevalent in $44 \%$ of the cases, followed by the following: Down Syndrome 18\%, Autistic Spectrum Disorder 12\%, Myelomeningocele 12\%, Deafness 3\%, Diabetes 3\%, Prader Willi Syndrome 3\%, Moebius Syndrome 3\% and Lenox Syndrome 3\%. Concerning to oral condition, $44 \%$ showed a poor oral condition, $32 \%$ deficient and $24 \%$ satisfactory. Conclusion: Most of the children were male, 8 years old, from Amazonas inland cities, with neurological deficiency and poor oral condition, suggesting that the presence of the dental surgeon composing the multidisciplinary hospital team helps to outline a preventive and curative treatment plan suitable for these individuals.
\end{abstract}

Descriptors: Disabled people; hospital dentistry; oral condition. 


\section{INTRODUÇÃO}

Pessoas com deficiência (PcD) são aquelas que apresentam uma condição ou alteração, seja ela simples ou complexa, por um curto período de tempo ou por toda sua vida. Além disso, a alteração ou condição pode ser de etiologia mental, física, biológica, social e/ou comportamental. Em razão desse quadro completo, as PcDs necessitam de uma abordagem especial, multiprofissional e de um protocolo especifico de atendimento ${ }^{1}$.

Em se tratando da terminologia utilizada para nomear essas pessoas, diversas nomenclaturas já foram designadas aos pacientes com deficiência, como pacientes com necessidades especiais, pacientes portadores de deficiência, pessoas excepcionais, pacientes especiais, inválidos, incapazes, entre outras. Entretanto, em 2008, houve a primeira Convenção de Direitos da Pessoa com Deficiência, na qual foi aprovado, por meio do Decreto Legislativo no 186/2008, o texto que altera o termo 'pessoa portadora de deficiência' para 'pessoa com deficiência' - (PcD), pela Portaria 2.344/2010 da Secretaria dos Direitos Humanos ${ }^{2,3}$.

Cerca de $10 \%$ da população mundial é constituída por Pessoas com Deficiência (PcD). No Brasil, há cerca de 160 milhões de habitantes e estima-se que 23,9\% possuem algum tipo de deficiência4. No que concerne ao Amazonas, nos últimos dez anos, o estado teve um aumento do número de deficientes de 96,8\%, e, segundo o Instituto Brasileiro de Geografia e Estatísticas (IBGE), em 2010, existiam 790.647 PcDs. A capital do estado, Manaus, apresentava, neste censo, cerca de $25,6 \%$ pessoas com algum tipo de deficiência 5 .

0 atendimento odontológico para pacientes considerados com deficiência pode ser difícil, pois são pessoas que, em sua maioria, não têm habilidade para realizar de forma satisfatória, uma higiene oral e, ainda, muitos não permitem que outras pessoas a façam, dificultando assim a higienização. Somam-se a isso, uma alimentação que pode ser pastosa e/ou cariogênica, e uso de medicamentos em forma de xaropes. Há de se salientar, ainda, que o comportamento pode ser imprevisível ou ainda agressivo, e por último, não raras vezes, há um alto uso de fármacos ${ }^{6,7}$.

A forma de abordagem e o tratamento odontológico de pacientes com deficiência envolvem a compreensão de dificuldades, como as motoras, o grau de limitação física e as dificuldades de comunicação. 0 conhecimento do perfil e o diagnóstico de pacientes atendidos e internados são fundamentais para minimizar os problemas bucais da população ${ }^{8}$.

A Odontologia Hospitalar tem como principal foco o cuidado de pacientes hospitalizados que necessitam de tratamentos especiais, buscando ações preventivas, diagnósticas, terapêuticas e paliativas, e possíveis complicações, através da higiene bucal sistemática. A abordagem requer uma equipe multiprofissional de alta complexidade, operando tecnologias que possam tornar melhor ou prolongar a vida do paciente internado $0^{9,10}$.

É substancial que haja a inserção de uma equipe multidisciplinar em ambiente hospitalar, visando a uma reabilitação e melhora no quadro de saúde dos pacientes, visto que estes, em sua maioria, apresentam também problemas sistêmicos ${ }^{11}$.

Durante a internação, pacientes com deficiência estão sujeitos a situações diferentes de rotina, começando pela estadia em um ambiente diferente do habitual. Podem responder de uma maneira diversificada, tanto comportamental como psicológica, podendo gerar ansiedade e estresse para os mesmos, como também para pais e familiares. A fragilidade sistêmica de um paciente internado está correlacionada ao desenvolvimento de patologias e fatores determinantes, como a cárie e as doenças periodontais durante o período de internação8,12.

Habitualmente, os responsáveis e/ou cuidadores não são orientados sobre como realizar a correta higienização após a ingestão de medicamentos e esta tarefa se torna difícil na rotina hospitalar, frisando a necessidade de um cirurgião-dentista na equipe ${ }^{12}$.

Desta forma, este estudo teve como objetivo traçar o perfil dos pacientes com deficiência internados no Instituto de Saúde da Criança do Amazonas (ICAM). 


\section{MATERIAL E MÉTODO}

Tratou-se de um estudo exploratório, observacional do tipo transversal, realizado com pacientes com deficiência atendidos pelo Instituto de Saúde da Criança do Amazonas (ICAM), na cidade de Manaus, estado do Amazonas. Estes foram submetidos à análise do perfil durante o período de fevereiro a abril de 201911 , tendo sua aprovação pelo CEP de número: 2.909.687.

Os responsáveis que concordaram em participar do estudo assinaram o Termo de Consentimento Livre e Esclarecido (TCLE), sendo esse o critério de inclusão. Foram excluídos os pacientes cujos responsáveis não aceitaram a participação na pesquisa.

Este estudo analisou 34 pacientes, que tiveram seus dados coletados dos prontuários médicos relativos a gênero, a faixa etária, o estado de origem e a patologia de base de cada indivíduo. Além disso, a condição de saúde bucal também foi avaliada, por um único avaliador previamente calibrado, utilizando o equipamento de proteção individual (EPI) completo, luz indireta (lanterna), espátula de madeira, espelho e cotonete.

Para avaliar a condição de saúde e higiene bucal, foi observada e registrada a presença de biofilme, saburra, halitose, gengivite, restos alimentares e sangramentos ${ }^{13}$. A higiene satisfatória se dava com a ausência de todos os itens ou apenas um destes; a higiene deficiente, quando ocorresse a presença de dois ou três; e por último, uma higiene precária, quando houvesse a presença de quatro ou mais itens. Uma ficha foi desenvolvida para registrar os dados obtidos. (Tabela 1).

Tabela 1. Ficha da avaliação da condição bucal

NÚMERO DO PRONTUÁRIO:

IDADE:

PROCEDÊNCIA:

MANAUS ( ) INTERIOR DO AMAZONAS ( ) OUTROS ( )

GÊNERO:

MASCULINO ( ) FEMININO ( )

PATOLOGIA BASE:

CONDIÇÃO DE SAÚDE BUCAL:

( ) BIOFILME

( ) SABURRA

( ) HALITOSE

( ) GENGIVITE

( ) RESTOS ALIMENTARES

( ) SANGRAMENTOS

() SATISFATÓRIO () DEFICIENTE ( ) PRECÁRIA

Fonte: Elaboração própria para anotação dos dados relativos ao estudo.

Os dados obtidos tiveram sua análise dispostas em figuras e tabelas, utilizando o programa Excel® 2013 e Word ${ }^{\circledR} 2013$.

\section{RESULTADO}

Dos 34 pacientes com deficiência avaliados, houve prevalência do gênero masculino com $70 \%$ de pacientes e 30\%, feminino. Os dados relativos à faixa etária variaram de 1 a 15 anos e mostraram que predominou a faixa etária de 5 a 10 anos, com a idade de 8 anos mais frequente. No que diz respeito à procedência, os resultados revelaram um percentual de $60 \%$ para cidades do interior do Amazonas e 40\% para a capital (Tabela 2). 
Tabela 2. Pacientes internados no ICAM, quanto ao gênero, idade e procedência

\begin{tabular}{lcc}
\hline \multicolumn{1}{c}{ Características } & n & Frequência Relativa (\%) \\
\hline Sexo & 24 & \\
Masculino & 10 & $70 \%$ \\
Feminino & & $30 \%$ \\
Faixa Etária & 7 & $20 \%$ \\
0 a 4 & 19 & $56 \%$ \\
5 a 10 & 8 & $24 \%$ \\
11 a 15 & & \\
Procedência & 14 & $40 \%$ \\
Manaus- AM & 20 & $60 \%$ \\
Interior do Amazonas & & \\
\hline
\end{tabular}

Fonte: Dados da pesquisa.

Conforme pode ser observado na Tabela 3, no que tange à patologia base, a Doença Neurológica teve prevalência em 44\% dos casos, seguida da Síndrome de Down com 18\%, do Transtorno de Espectro Autista (TEA) com 12\% e, da Mielomeningocele com 12\%. Cada uma das deficiências - Surdez, Diabetes, Síndrome de Prader Willi, Síndrome de Moebius e Síndrome de Lennox-Gastaut - com 3\% dos pacientes com deficiência internados.

Tabela 3. Distribuição das patologias base

\begin{tabular}{lcc}
\hline \multicolumn{1}{c}{ Patologia Base } & n & Frequência Relativa (\%) \\
\hline Doenças Neurológicas & 15 & $44 \%$ \\
Síndrome de Down & 6 & $18 \%$ \\
TEA & 4 & $12 \%$ \\
Mielomeningocele & 4 & $12 \%$ \\
Surdez & 1 & $3 \%$ \\
Diabetes & 1 & $3 \%$ \\
Síndrome de Prader Willi & 1 & $3 \%$ \\
Síndrome de Moebius & 1 & $3 \%$ \\
Síndrome de Lennox- Gastaut & 1 & $3 \%$ \\
\hline
\end{tabular}

Fonte: Dados da pesquisa.

No que diz respeito à condição da saúde bucal dos pacientes com deficiência internados, tivemos como resposta que $24 \%$ apresentaram condição bucal satisfatória, $32 \%$ deficiente e, $44 \%$ precária (Tabela 4).

Tabela 4. Condição Bucal dos pacientes internados no ICAM

\begin{tabular}{ccc}
\hline Condição Bucal & n & Frequência Relativa (\%) \\
\hline Satisfatória & 8 & $24 \%$ \\
Deficiente & 11 & $32 \%$ \\
Precária & 15 & $44 \%$ \\
\hline
\end{tabular}

Fonte: Dados da pesquisa.

\section{DISCUSSÃO}

O estudo do perfil dos pacientes com deficiência pode gerar dados relevantes ao conhecimento da comunidade científica e da sociedade, bem como registrá-los nacionalmente. Assim, pode-se em médio prazo aumentar a visibilidade desses pacientes no que diz respeito às políticas de 
saúde, cujas medidas permitirão a execução de ações específicas e direcionadas para essa parcela da população.

Neste estudo, a maioria dos pacientes foi do gênero masculino, corroborando com a maioria dos estudos já publicados na literatura tanto no Brasil quanto no exterior ${ }^{12-16}$, embora seja divergente do estudo de Kohata et al. ${ }^{17}$, o qual revelou ter o sexo feminino como mais prevalente.

A faixa etária encontrada variou de 2 a 15 anos. Isto se explica por ser um hospital pediátrico, englobando crianças e adolescentes, e tal fato corrobora com o estudo de Veríssimo et al. ${ }^{18} \mathrm{~A}$ média de idade mais frequente foi a de 8 anos, como também pode ser visto no estudo de Silva et al. ${ }^{19}$

Em relação à procedência, a maioria dos pacientes era proveniente do interior do Amazonas e o restante era da capital, ratificando com o estudo de Silva et al. ${ }^{19} \mathrm{~A}$ maioria destes pacientes veio para a capital para conseguir atendimento talvez pela falta de infraestrutura da área de saúde e pela carência de atendimentos especializados no interior do estado, concordando com Roselino et al. ${ }^{20}$, que relatam que o acesso aos serviços de saúde é diretamente influenciado pela condição social das pessoas e pelo local em que habitam.

A patologia base mais prevalente neste estudo foram as doenças neurológicas (deficiência mental, paralisia cerebral ou anomalias congênitas associadas a deficiência mental), concordando com os achados de Previtali et al. ${ }^{14}$, Santos et al..$^{21}$, Gondim et al. ${ }^{22}$, Bizarra et al. ${ }^{23}$, diferindo de outros trabalhos como Kohata et al. ${ }^{17}$, Silva et al. ${ }^{19}$ e Mohamed Rohani et al. ${ }^{24}$, que revelam que a maioria foi de distúrbios comportamentais, sistêmicos ou genéticos.

A Síndrome de Down foi a segunda patologia mais encontrada, reafirmando os achados de outros estudos já realizados ${ }^{19,21,23}$, em que a deficiência mental, a paralisia cerebral e a Síndrome de Down foram as condições mais frequentes, discordando de Veríssimo et al. ${ }^{18}$ e Sachdeva et al. ${ }^{25}$, em que estiveram presentes nos achados as doenças sistêmicas crônicas.

Os dados encontrados sobre a condição bucal estão de acordo com o estudo de Veríssimo et al. ${ }^{18}$, no qual a maioria é classificada como precária/insatisfatória, sendo justificada pela falta de capacidade desses pacientes para um melhor desempenho dos procedimentos necessários à remoção mecânica da placa bacteriana. Uma higienização deficitária pode ter efeitos e impactos na qualidade de vida, afetando negativamente o bem-estar geral. Em algumas deficiências, hábitos simples podem se tornar difíceis, como a higiene bucal, tendo em vista que muitos não possuem habilidades e não permitem que outras pessoas a façam ${ }^{22}$.

Pode-se notar a prevalência da condição ruim nas pesquisas de Gondim et al. ${ }^{22} \mathrm{e} \mathrm{Lima} \mathrm{et} \mathrm{al.12,}$ contrastando com a pesquisa de Costa et al. ${ }^{13}$, em cujo estudo a condição deficiente foi a mais encontrada. Pacientes que apresentam de fato uma insuficiência motora têm sua higienização negligenciada pelos cuidadores. A conscientização de familiares e dos cuidadores é de extrema importância para a manutenção da saúde bucal, pois a dificuldade em manter hábitos favoráveis de higiene bucal é um fator de risco de cárie em pacientes com deficiência. A partir de dados, é possível classificar a condição bucal do paciente em satisfatória/boa, deficiente e precária/ruim $^{22,25}$.

De acordo com a literatura, o conhecimento do perfil de pacientes com deficiência pode melhorar a qualidade do planejamento dos cuidados clínicos oferecidos e pode, em estudos seguintes, facilitar a construção de protocolo de atenção a essa população ${ }^{14}$.

\section{CONCLUSÃO}

A maioria das crianças era do gênero masculino, na faixa etária de 5 a 10 anos, com a idade de 8 anos mais frequente, procedentes do interior do Amazonas, com deficiência neurológica e condição bucal precária. A presença do cirurgião-dentista compondo a equipe multidisciplinar hospitalar pode auxiliar a traçar um plano de tratamento preventivo e curativo adequado a esses 
indivíduos, uma vez que pacientes pediátricos com deficiência necessitam de cuidados odontológicos, principalmente quando se encontram hospitalizados. Com isso, os achados obtidos podem orientar uma conduta que forneça uma base para que se melhorem os serviços.

\section{AGRADECIMENTOS}

Agradeço ao Instituto de Saúde da Criança do Amazonas (ICAM), por fornecer os dados, a infraestrutura e os meios para a realização deste artigo. À minha orientadora e à co-orientadora, pela atenção e disposição.

\section{REFÊRENCIAS}

1. Brasil. Ministério da Saúde. Secretaria de Atenção à Saúde. A saúde bucal no sistema único de saúde. Brasília: Ministério da Saúde; 2018. p. 251-282.

2. Brasil. Decreto no 186 de 09 de julho de 2008. Diário Oficial da União [Internet]. Brasília, 2008 [citado em 2020 Ago 4]. Disponível em: http://www.planalto.gov.br/ccivil_03/constituicao/Congresso/DLG/DLG-1862008.htm

3. Alencar L, Amorim DG. A viabilização da inclusão de pessoas com deficiência ao mercado de trabalho. Revista Opara. 2014 Jan-Dez;4(1):1-12.

4. Brasil. Secretaria de Direitos Humanos da Presidência da República. Secretaria Nacional de Promoção dos Direitos da Pessoa com Deficiência. Coordenação-Geral do Sistema de Informações sobre a Pessoa com Deficiência. Cartilha do Censo 2010: pessoas com deficiência. Brasília: SDH-PR/SNPD; 2012.

5. Garcia V. Número de pessoas com deficiência quase dobra e atinge $23 \%$ no Amazonas [Internet]. Deficiente Ciente - 0 blog da Inclusão e Cidadania; 2012 [citado em 2020 Ago 4]. Disponível em: https://www.deficienteciente.com.br/numero-de-pessoas-com-deficiencia-quase-dobra-e-atinge-23-noamazonas.html

6. Christensen GJ. Special oral hygiene and preventive care for special needs. J Am Dent Assoc. 2005 Aug;136(8):1141-3. http://dx.doi.org/10.14219/jada.archive.2005.0319. PMid:16161370.

7. Moterle C, Miranda F, Cavalheiro S, Faccin M, Garrastasu MD, Costa MMTM. Promoção de saúde bucal em pacientes com necessidades especiais: relato de experiência do curso de odontologia da UNOESC Joaçaba. Ação Odonto. 2017(1):1-2.

8. Menezes TOA, Smith CA, Passos LT, Pinheiro HHC, Menezes SA. Perfil dos pacientes com necessidades especiais de uma clínica de odontopediatria. Rev Bras Promoç Saúde. 2011 Jan-Jun;24(2):136-41.

9. Marín C, Nunes dos Santos MH, Rabaldo Bottan E. Dental surgeons' perceptions of hospital dentistry. Rev Bras Odontol. 2017;74(1):14-7. http://dx.doi.org/10.18363/rbo.v74n1.p.14.

10. Andrade E, de Souza LGS, Teodoro MKR, Gominho LF, Fonseca G Jr. Assistência odontológica a pacientes de unidade de terapia intensiva (UTI). The Open Brazilian Dentistry Journal. 2020;1(1):1-11.

11. Oliveira ALP, Chagas SKM, Peixoto MOB, Peixoto FB, Cruz VSA, Ferreira SMS, et al. Avaliação sociodemográfica e odontológica de pacientes com deficiência atendidos na clínica-escola de uma instituição de ensino superior. RvAcBO. 2017;26(2):58-64.

12. Lima MCPS, Lobo INIR, Leite KVM, Muniz GRLM, Steinhauser HC, Maia PRM. Condição de saúde bucal de crianças internadas no Hospital Municipal Infantil de Imperatriz-Maranhão. Rev Bras Odontol. 2016 JanMar;73(1):24-9. http://dx.doi.org/10.18363/rbo.v73n1.p.24.

13. Costa DC, Saldanha KFD, de Sousa AS, Gaetti-Jardim EC. Perfil de saúde bucal dos pacientes internados no Hospital Universitário Maria Aparecida Pedrossian, Campo Grande (MS). Arch Health Invest. 2016;5(2):707. http://dx.doi.org/10.21270/archi.v5i2.1301. 
14. Previtali EF, Ferreira MCD, Santos MTBR. Perfil dos pacientes com necessidades especiais atendidos em uma Instituição de Ensino Superior. Pesqui Bras Odontopediatria Clin Integr. 2012 Maio;12(1):77-82. http://dx.doi.org/10.4034/PBOCI.2012.121.12.

15. Limeres J, Martínez F, Feijoo JF, Ramos I, Liñares A, Diz P. A new indicator of the oral hygiene habits of disabled persons: relevance of the carer's personal appearance and interest in oral health. Int J Dent Hyg. 2014 May;12(2):121-6. http://dx.doi.org/10.1111/idh.12033. PMid:23730898.

16. Chang J, Lee JH, Son HH, Kim HY. Caries risk profile of Korean dental patients with severe intellectual disabilities. Spec Care Dentist. 2014 Jul-Aug;34(4):201-7. http://dx.doi.org/10.1111/scd.12047. PMid:25039380.

17. Kohata VKG, Zacarias RP Fo, Alves AO Fo, Medina PO, Hanan SA. Profile of patients with special needs assisted at a brazilian university. Pesqui Bras Odontopediatria Clin Integr. 2019;19(1):e4057. http://dx.doi.org/10.4034/PBOCI.2019.191.37.

18. Veríssimo AH, Azevedo ID, Rêgo DM. Dental profile of special needs patients in a pediatric hospital of a Brazilian public university. Pesqui Bras Odontopediatria Clin Integr. 2013 Out-Dez;13(4):329-35. http://dx.doi.org/10.4034/PBOCI.2013.134.05.

19. Silva ZCM, Pagnoncelli SD, Weber JBB, Fritscher AMG. Avaliação do perfil dos pacientes com necessidades especiais da clínica de odontopediatria da faculdade de odontologia da PUCRS. Rev Odonto Ciênc. 2005 OutDez;20(50):313-8.

20. Roselino PL, Damasceno JL, Figueiredo GLA. Saúde bucal na atenção primária à saúde: articulações entre o ensino e a estratégia de saúde da família. Rev Odontol UNESP. 2019;48:e20190081. http://dx.doi.org/10.1590/1807-2577.08119.

21. Santos CML, Falcão MML, Souza ALD, Santos MS, Coelho AA. Perfil epidemiológico dos pacientes com necessidades especiais atendidos em um centro de especialidades odontológicas do interior baiano. Rev Baiana Saúde Pública. 2014 Jan-Mar;38(1):83-94. http://dx.doi.org/10.5327/Z0100-02332014380100014.

22. Gondim LAM, Andrade MC, Maciel SSSV, Ferreira MAF. Perfil epidemiológico das condições dentárias e necessidade de tratamento dos portadores de deficiência da cidade de Caruaru, Pernambuco, Brasil. RGO. 2008 Out-Dez;56(4):393-7.

23. Bizarra MF, Graça SR. 0 perfil do paciente da consulta para pessoas com necessidades especiais da FMD-UL. Rev Port Estomatol Med Dent Cir Maxilofac. 2010 Apr-Jun;51(2):69-74. http://dx.doi.org/10.1016/S16462890(10)70089-3.

24. Mohamed Rohani M, Calache H, Borromeo GL. Referral patterns of special needs patients at the Royal Dental Hospital of Melbourne, Victoria, Australia. Aust Dent J. 2017 Jun;62(2):173-9. http://dx.doi.org/10.1111/adj.12465. PMid:27670933.

25. Sachdeva SK, Raj SS, Kaushik A, Sharma D, Verma K, Dutta S, et al. Prevalence of medical problems among patients attending a dental school in India - a cross sectional study. OHDM. 2015;14(6):429-34.

\section{CONFLITOS DE INTERESSE}

Os autores declaram não haver conflitos de interesse.

\section{*AUTOR PARA CORRESPONDÊNCIA}

Sara Maria Araujo do Nascimento, Av. Carvalho Leal, 1777, Cachoeirinha, 69065-001 Manaus - AM, Brasil, e-mail: sara_araujo38@hotmail.com

Recebido: Setembro 2, 2020

Aprovado: Outubro 2, 2020 